\title{
AN INJECTING FLUID FOR PRESERVING CADAVERS FOR DISSECTION.
}

\author{
BY \\ WILLIAM C. LUSK. \\ From the Anutomical Department of the New York University and Bellevue \\ Hospital Hedical College.
}

Experimental work on the preservation of cadavers for dissection has been done in this country by Keiller, ${ }^{1} \mathrm{Mall}^{2}$ and Souchon. ${ }^{3}$

Keiller uses a preserving fluid containing formalin, carbolic acid and glycerin. Mall uses carbolic acid, glycerin and alcohol. Souchon's formula for embalming bodies for the dissecting-room comprises arsenious acid, formalin, carbolic acid, glycerin, alcohol, and creosote. In here voicing the use of carbolic acid, it is of interest to recall that the valuable deodorant and preservative properties of this chemical in the preservation of cadavers were first recognized by Rüdinger in the early seventies, and as well that carbolic acid has been used continuously at the Johns Hopkins since the opening of the Medical School in 1893.

During the years 1899 to 1902 , when the writer had charge of the dissecting-room at the New York University and Bellevue Hospital Medical College, he made experimental studies on the preservation of the cadavers for dissecting-room work, with the intent of producing a material that would possess firmness with pliability, and that would not readily decompose or dry up during dissection. The following formula was the finished product of the experimentation, which filled all the requirements aimed for, and besides, when formalin was not added, preserved the bright red color of the muscles. The addition

${ }^{1}$ Keiller. Phila. Med. Journ., 1900, p. 1248. Amer. Jour. Anat., II, 1902-03, Proceedings Assoc American Anatomists, p. vil.

'Mall. Johns Hopkins Hospital Bulletin, XVI, February, 1905.

"Souchon. Anatomical Record, II, 1908. p. 244. 
of the two-thirds per cent. of formalin darkened the muscles.somewhat.



The solution of hydrosodium arsenite is made as follows:

Arsenious acid (about 97 per cent pure) ......50 ounces.

Sodium carbonat. siccum ..............40 ounces.

Water $\ldots \ldots \ldots \ldots \ldots \ldots \ldots \ldots \ldots \ldots \ldots \ldots \ldots \ldots$ 10,000 c.c.

Boil from two to three hours.

Bodies of 100 pounds require for preservation from 4000 to 5000 cc. of the injecting fluid, and for each 25 pounds additional weight of the body there should be added about 1000 cc. of the fluid.

This injecting fluid, or one made in all essentials like it, has since been used in the college dissecting-room for the past six years with eminent satisfaction. The fluid produces the best material for dissection when the instructions of the formula are adhered to with exactness. The use of carbolic acid as a preservative for anatomical material has the particular advantage that the bodies injected with it do not readily decompose, and consequently the atmosphere of the dissecting-room is not loaded with the gases of decomposition and there need be no unpleasant odor about the premises. The glycerin in the injection possesses the advantages, first, that in mixing the fluid it causes a ready solution of the carbolic acid, and second, after 
iujection into the body, it prevents rapid drying of the tissues. By its hygroscopic property it will take up sufficient moisture simply from a dampened fabric to keep the structures soft, thus doing away with any need for drenching the dissection with water, which procedure only dissolves out the chemical preservatives and thus aids decomposition.

Carbolic acid and glycerin in combination with alcohol in varying proportions are much used by medical schools for embalming cadavers. The writer in experimenting with this mixture found that while the above mentioned desirable properties of the carbolic and the glycerin held good, yet certain disadvantages were apparent. Thus the carbolic browned or blanched the muscle fibers, and seemed to destroy their tensile strength, making them readily frayable when picked with forceps. Also when used in the considerable strength of 33 per cent. the carbolic would numb the fingers of the dissector. The glycerin in this combination seemed to be the cause, probably from its hygroscopic action, of making the tissues, particular'y the dependent ones, soggy with water.

The question arose how to utilize carbolic acid and glycerin in an embalming fluid in a way to reap their advantages and at the same time eliminate their objectionable features. The addition to a mixture of glycerin, carbolic acid and alcohol, of a solution of hydrosodium arsenite with a small excess of sodium carbonate in the proportions given in the above formula, finally gave the desired result. The wetting action of the glycerin on the tissue was offset so that the latter became quite free from excessive moisture, the muscles were firm and red, and the fasciæ of a normal tensile strength. The viscera were pliable and well preserved. The cut tissues of a freshly injected body would emit a slightly sourish odor which was characteristic of a well made injection.

The thorough mixing of the ingredients and the letting of the solution stand for 12 to 24 hours before use, are very important elements in securing the best result. The necessity for the thorough mixing is particularly for the purpose of securing an equable distribution in the fluid, of the carbolic acid which, if not evenly mixed, will produce brownish areas in the injected muscles. The formalin too when used should be well mixed since there is a very small quantity of it to be diffused throughout a large volume of fluid. 
In explanation of the better result from letting the injecting fluid stand for some hours after mixing, the writer should state it as his belief that a chemical combination takes place between the carbolic acid in solution and the excess of soda carbonate, to form a carbolate of soda, and that a certain length of time for the complete conversion of the carbolic acid is required, so that if the solution is injected immediately after it is mixed, this change will not have had time to have fully taken place, and the unchanged carbolic acid then exercises its corrosive effect on the muscles. Whereas, with the carbolic acid converted by the alkali into the carbolate of soda, while retaining its power as a preservative, its ill effects on the muscles are counteracted. That a chemical change does take place between a mixture of glycerin, carbolic acid and alcohol, and a solution of sodium carbonate, is evidenced by the evolution of gas which takes place when these two liquids are mixed together. In further evidence that an alkali does in some way offset a destructive action on muscle fibers attributable to carbolic acid, it was found by comparing one body injected with a preserving fluid consisting of carbolic acid, glycerin and alcohol, made slightly alkaline with potassium carbonate, with another body injected with the same chemicals but made more strongly alkaline with the potassium carbonate, that in the latter instance the muscle fibers were much stronger than in the former. It was also noted in the course of experimentation that where the injected muscle fibers had an alkaline reaction to litmus paper they were much firmer and less easy to fray when picked with forceps, than where the reaction was acid. In this connection it is an interesting fact that the reactions of both carbolic acid and glycerin are alkaline, while that of aicohol is acid as is also that of the tissues of the dead body. Thus it might be regarded possible for an alkaline reaction of the muscles to be brought about from the carbolic acid and glycerin in the injecting fluid, yet as a matter of fact when glycerin, carbolic acid, alcohol and hydrosodium arsenite were used in combination, the tissues seemed n€ver evenly alkaline throughout the entire body unless a sufficient amount of sodium carbonate had been added in the making up of the fluid.

The need for letting the fluid stand before use after the formalin had been added, was demonstrated by the resulting greater toughening 
of the fascir and skin and the hardening of the fat, which were not so apparent when this regulation was not observed.

In making up the injecting solution corresponding to the above formula, since glycerin is a ready solvent of carbolic acid, these two ingredients are first mixed together, the necessary flagellation of the liquids to effect the solution of the carbolic being best done with an egg-beater.

The necessity for the alcohol arises from the fact that this fluid thins the glycerin down so that the injection will flow freely from a reservoir through the arteries into the tissues of the cadaver. Also unless the amount of alcohol in the above formula equals that of the glycerin, the injecting fluid will be too thick to pass entirely out of the terminal arterial branches and, lodging in these, will obstruct the entrance into them of the starch injection.

The hydrosodium arsenite, besides being an excellent preservative, seemed to be the factor which enabled the muscles to retain their $r \in d$ color in the presence of the carbolic acid and which offset the apparent action of the glycerin to make the tissues soggy. The hydrosodium arsenite was made by boiling arsenious acid $\left(\mathrm{As}_{2} \mathrm{O}_{3}\right)$ with sodium carbonat. siccum $\left(\mathrm{Na}_{2} \mathrm{CO}_{3}\right)$ or dry soda ash, in water for two or three hours. The ordinary carbonate of soda or washing soda, cuntaining ten molecules of water of crystallization for each molecule of the salt, and therefore representing less of the salt than an equal weight of the dry soda ash, if used, should be calculated accordingly. On boiling the arsenious acid and soda together in water, it was found, estimating by atomic weights, that the acid would actually combine with but about one-third the quantity of soda that would be necessary to completely convert it into a triple sodium arsenite, so that the resulting product would be a hydrosodium arsenite, probably having for the most part the formula $\mathrm{H}_{2} \mathrm{NaAsO}_{3}$. Thus it was found that about 14 ounces of dry soda ash would combine with 31 ounces of arsenious acid (or in the proportion of 22.6 to 50 ) to make a solution of hydrosodium arsenite having just the slightest possible amount of alkali in excess, or practically making a neutral solution. The prolonged boiling for from two to three hours was necessary to effect the combination of the greatest possible amount of 
soda with the arsenious acid. The proportion of sodium carbonate used in the injecting fluid seemed to have a threefold influence, first, on the amount of moisture in the injected tissues, second, on the power of the arsenic to irritate the hands, and third, on the strength of the muscle fibers. Bodies injected with a nearly neutral hydrosodium arsenite solution in combination with glycerin, carbolic acid and alcohol were noticeable for the absence of all sogginess of the tissues. The structures were dry and pliable and the color of the muscles was red, but there were two objections; - first, the muscle fibers were weaker than when more of the alkali was used, and second, especially objectionable was the severe arsenic irritation beneath the finger nails of those dissecting the bodies thus injected. The addition of a small excess of the sodium carbonate beyond what would combine with the arsenious acid solution, now remedied these two difficulties, the proportion used being sodium carbonat. siccum 40 ounces to arsenious acid 50 ounces. The tensile strength of the injected muscle fibers in the presence of this small excess of carbonate of soda is very much greater than that of living muscle fibers, the picking of the injected fibers with a thumb forceps requiring some considerable force to rupture them. In explanation of this the writer would state as his belief that the result is partly due, as already mentioned, to a transformation of carbolic acid into a carbolate of soda in which combination the carbolic supposedly no longer exerts its destructive action on the muscles, and also there may be besides a strengthening influence on the muscles, produced by the hydrosodium arsenite in the presence of an alkaline medium. With this added amount of carbonate of scda also the arsenic irritation beneath the finger nails was practically done away with. It was of interest to note that a considerable excess of sodium carbonate added to the injecting fluid, would reverse the evident drying action of the more nearly neutral solution, causing the tissues to be again very moist, so that for the sake of getting as dry a tissue as practicable, only enough soda to prevent finger irritat1on by the arsenic was used, which amount was found to be a little more than that needed to cause strengthening of the muscles.

An arsenate of soda, alkaline with sodium hydrate, was tried in combination with glycerin, carbolic acid and alcohol, the effect of 
which seemed to be to produce generally wet tissues, and muscle fibres weak in consistence though of a good red color.

By the addition of the two-thirds per cent of formalin (one-fourth per cent formaldehyd) to the formula here advocated, it seemed particularly that the fascix were strengthened, the skin toughened, and the fat hardened. The muscles were darkened a little. Since formalin is evanescent it cannot have a long continued action as a preservative, so that the main purpose for its use would be the primary arrest of decomposition in green bodies. The formalin is however really not a necessary addition to the injecting fluid, since green bodies, many days dead, were injected without the formalin having been added and the injected tissues have shown a perfect arrest of decomposition and possessed the qualities desirable for good dissecting material. However, formalin is such a popular disinfectant for the arrest of decomposition and is so much used for the purpose of preserving anatomical and pathological specimens, that it is mentioned here to specify in what strength it can be used to the best advantage in connection with the injecting fluid here described. If formalin of a greater strength than about two-thirds per cent be added the fasciæ and skin will be found to have been unduly toughened so as to materially add to the labor of dissecting. It was noteworthy in one cadaver where an injection containing a considerable strength of formalin (probably 10 per cent) in combination with an arsenic salt was tried, that the muscles were made stiff, and at the same time lost all tensile strength, so that when the arms were raised upward the pectoral muscles tore across like paper. This is in keeping with the experience of Addison $^{4}$ who, in advocating an injecting fluid comprising formalin, arsenic and potassium carbonate, the formalin being used in a strength of 5 per cent for its hardening effect on the body, notes that the muscles by this combination are "made brittle and are torn across in putting the body in the lithotomy position unless care be exercised." He adds, "For demontrating the various fascial planes and their attachments the hardened subject is excellent."

The preserving fluid herein described is readily injected into the bodies by means of a percolator, that is, a reservoir hung four or five

‘Addison. Quart. Med. Journ., Sheffield, 1897-8, vI, p. 251. 
feet above the body with a tube connection to convey the fluid into an artery. The right carotid was always used.

After having injected the preserving fluid, fully twenty-four hours should be let pass before injecting the arteries with starch. It was found that a gelatin injection into the arteries would not stiffen after the use of this injecting fluid.

For storing the embalmed bodies, the writer, toward the end of his period of service in the anatomical department, became interested in the method employed by Shepherd ${ }^{5}$ at McGill University, and te:sted it sufficiently to demontrate that it had a probable value above that of cold storage. The method consists in placing the bodies in boxes which can be shut absolutely air-tight, and subjecting them therein to the fumes of alcohol deposited in the bottom of the box. The box should be lead-lined. The writer had the experience that a tighty jointed box made of two-inch plank and not lined, promptly cracked when the fumes of the alcohol were confined within it. Doctor Shepherd, in a recent communication, kindly extended to the writer the courtesy of here quoting from his personal experience with the alcohol method. Doctor Shepherd said: "In these closed tanks we have kept subjects for several years provided they are put in fresh -they neither dry nor mould and make capital subjects because in the dissecting-room they remain fresh."

During dissection the material injected as herein described keeps soft under sereral layers of damp fabric with all excess of moisture wrung out of the meshes. Too much water dissolves out the chemicals and macerates the tissue. If one desires to preserve the tissues particularly well during a dissection, or over a very long period of time, alcohol is then the best agent with which to moisten the part and to dampen the enveloping fabric. An impervious outer covering aids in preventing too rapid evaporation of the alcohol. To keep the fingers or toes, or other skin surface of one of these bodies exposed to the air from drying, wrap the part in gauze dampened in a solution containing about 10 to 15 per cent of glycerin and about 3 to 5 per cent of carbo'ic acid and then cover the whole with an impervious

'Slepherd. Proceedings Association of American Anatomists, May, 1900 , p. 23. 
covering such as oil cloth. The moisture will be retained for two or three weeks or more. The carbolic prevents maceration of the skin.

A body preserved with the above injection, if properly taken care of, can be dissected during the heat of summer without any offensive odor of decomposition.

Received for publication, December 14, 1908 . 